\title{
Cognitive Maps for Indirect Coordination of Intelligent Agents
}

\author{
Ioan SUSNEA ${ }^{1}$, Cristian AXENIE ${ }^{2}$
}

${ }^{1}$ University "Dunarea de Jos", 47, Domnească Street, Galati, 800008, Romania ioan.susnea@ugal.ro

2 Technical University of Munich, Arcisstrasse 21, Munich, 80333, Germany cristian.axenie@tum.de

\begin{abstract}
The core idea of the research described in this paper is that the processes of self-organization commonly called "Swarm intelligence" can be triggered by creating - through the collective effort of the whole population of agents - a „global cognitive map" derived from the interaction between the agents and the environment, and shared by all agents. We focus on multi-agent systems with human agents, and explore the technological means to automate the process of creating and sharing such cognitive maps, with the aim to induce controllable self-organization of the behaviour of the agents.
\end{abstract}

Keywords: multi-agent systems, cognitive maps, stigmergy, activity recognition, behaviour implicit communication.

\section{Introduction}

After several decades since Marshal McLuhan coined the term "global village" to describe the impact of the new computer and communication technology on our lives, there is a fast growing body of evidence that this impact goes far beyond any expectation.

We are a gregarious species, and the increase of the intensity of social interactions is linked to physical and physiological modifications of the brain ([8]). It seems that the Internet and the social networks literally change our physical brains, not just our habits.

Therefore, the true consequences of this unprecedented expansion of the communications in the Internet era are yet to be evaluated.
In this paper, we explore the inter-human communication from the perspective of multiagent systems (MAS), and self-organization.

One key concept for understanding the technologically mediated interactions between people is "to share". We share more than pictures and music preferences on Facebook: in a general sense, we share a common environment, which may include the IT infrastructure we use, and also any other physical, logical or cultural elements we can sense, and influence through our activity (see Figure 1).

This mechanism of indirect communication and coordination by means of traces created and sensed in a shared environment has been called "stigmergy". It was first described by Grasse

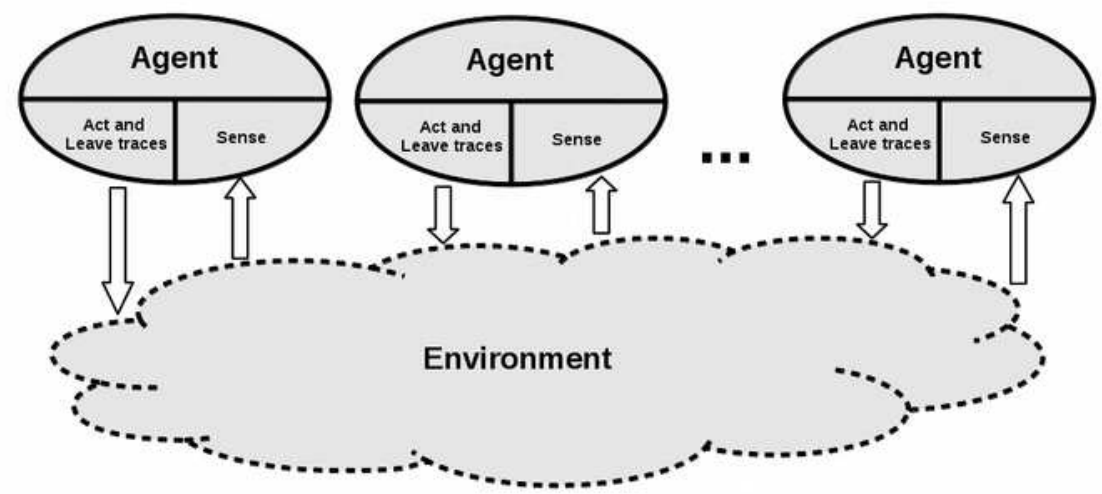

Figure 1. The core mechanism of stigmergy 
([9]), and later raised a great deal of interest as key factor of the "swarm intelligence" ([2]).

Stigmergy and swarm intelligence have been extensively studied in a wide variety of applications ([3]), mainly in robotics ([2], [3], [20]), and optimization problems ([7], 14]).

Valuable and extensive analyses of the mechanisms of stigmergic interactions in multiagent systems (MAS) are available in ([10], [18]).

In this paper, we discuss the stigmergic interactions between human agents from a slightly different perspective: we consider the case when the agents share not just a common environment, but also some mental representations thereof - cognitive maps.

The core idea of the research described here is that the processes of self-organization called „swarm intelligence" can be triggered by building - through the collective effort of the whole population of agents - a ,global cognitive map" stored in the distribution of traces created by the agents in the environment.

We outline the elementary operations and the technological means to automate the process of creating and sharing the collective cognitive maps in order to induce stigmergic behaviour in human MAS.

Apart from this introduction, the paper is organized as follows:

- Section 2 is a brief presentation of the conceptual framework that explains the connections between cognitive maps and stigmergy.

- In Section 3 we explore the possibility to automate the collective creation of shareable cognitive maps.

- Section 4 is reserved for conclusions.

\section{Cognitive Aspects of Stigmergy}

Regardless of the type of agents under consideration, from protozoa to humans, and beyond, to artefacts having a certain degree of autonomy, every action occurs in space. The space is "the recipient" where biologic agents find food sources, predators, partners for mating, shelter for themselves and their offspring, etc. Therefore, the way the agents perceive the space, the relative positions and distances between various elements thereof is crucial for survival, and determinant for the behaviour of the agents. Though some of the interactions between biological agents and the environment have been explained by simple mechanisms like phototaxis and chemotaxis, it is obvious that the majority of the spatial behaviour of most organisms cannot be reduced to such simple strategies. For this reason, Tolman ([25]) postulated the existence of certain neural structures called "cognitive maps" defined, in a general sense, as a mental model of the spatial environment.

Later, O'Keefe ([16]) provided a series of experimental data, and argued that there exist in a certain area of the brain (hippocampus) a class of cell specialized in processing the spatial information ("place cells"), which exhibit activity patterns obviously correlated with certain features of the space where the agent operates. Despite some critics, (a good review is available in [6]), the concept of "cognitive maps" is almost unanimously accepted, and has been explored from various perspectives: biology, psychology, engineering (robotics), cognitive sciences, architecture and urbanism, etc.

In engineering, the concept of cognitive maps has been mostly used in the context of robotics research. The approaches used here range from agents with simple, reactive behaviour, (where the cognitive map is implied by the existence of a localization systems e.g. odometry or GPS) to solutions wherein the agents build and maintain a complex global map of the environment ([26]).

A particularly interesting research direction has been suggested by Chialvo \& Millonas ([5]), who demonstrated, (using a probabilistic framework), that the self-organization processes called "swarm intelligence" ([2]) can be assimilated with building, through the activity of the whole population of agents, a global cognitive map, located not in the neural structures of the agents, but in the pheromone ([11]) traces created by the agents in the environment.

In fact, there is a striking similarity between the dynamics of sensing a pheromone distribution resulted from the activity of a swarm and a simple neural network. Apparently this was first suggested in our work ([20]).

Assuming that a generic ant senses the pheromone scent produced by $\mathrm{N}$ discrete sources, as shown in Figure 2, due to diffusion, the effect of the discrete pheromone sources is 
weighted by the distances between the sources and the sensing agent. Eventually, the superposition integrates the effects of all sources (see Figure 3).

Note the perfect match of the structure shown in Figure 3 with the well-known model of the artificial neuron (the Weber-Fechner law of perception adds a non-linear activation function to the effects of diffusion and superposition of the pheromones).

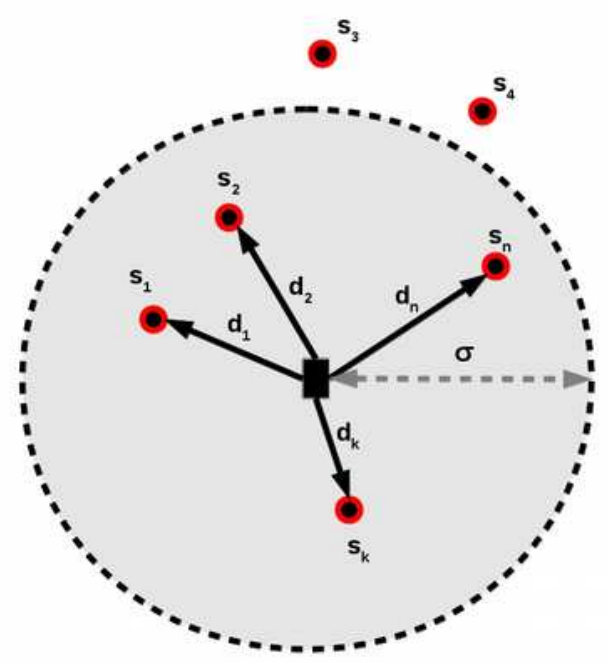

Figure 2. Notations used to describe the superposition of the effects of $\mathrm{N}$ discrete sources of pheromones

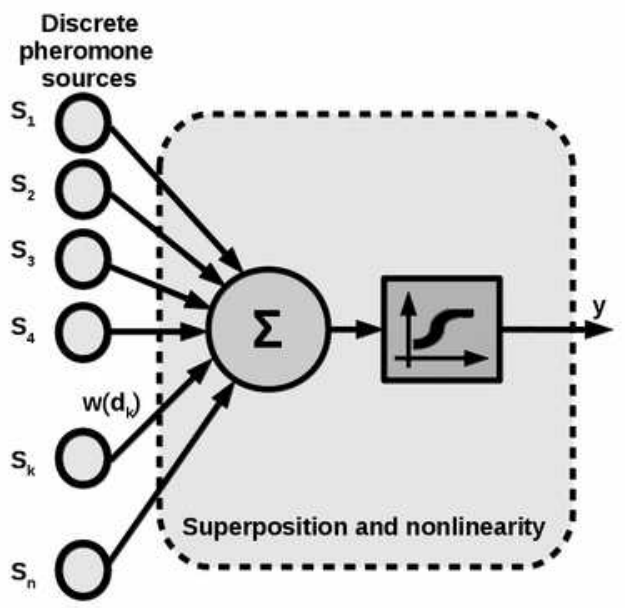

Figure 3. The process of sensing $\mathrm{N}$ discrete sources of pheromones is equivalent to the operation of a generic neuron

Biological agents like insects usually sense the pheromone traces by means of two antennas. Differential sensing provides the agents with valuable additional information about spatial gradients of the pheromone distribution.

We have used the equivalent neural network created this way (see Figure 4) to directly control a mobile robot for path following ([20]).

This neural connection between the agents and their environment suggests that, for each particular place of the environment, by simply sensing the pheromone traces, an agent instantly learns some information about the spatial distribution, and past behaviour of the other agents.

Assuming an instance capable to iterate the procedure of integrating this information for all the "places" of the environment, it is possible to obtain a global cognitive map of the respective environment.

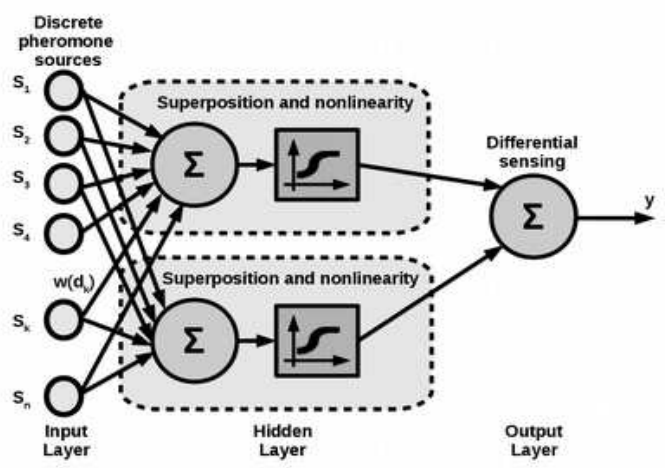

Figure 4. Differentially sensing pheromone traces with two antennas creates a small equivalent neural network

In the following section, we explore the possibility to create such an instance - a technological context that allows the (automated) creation of some data structures equivalent to global cognitive maps.

And, knowing the close connection between the cognitive maps and the behaviour of the agents, it can be presumed that sharing the resulting global maps with the agents can be a means to influence their behaviour, and induce self organization.

\section{Creating Global Cognitive Maps to Foster Stigmergic Interactions}

The current section introduces synthesis details of the proposed approach to build distributed maps enabling stigmergic interactions. 


\subsection{Virtual pheromones as the internal "scent" of cognitive maps}

We have made a first step towards building global cognitive maps in MAS by proposing the concept of "virtual pheromones", defined as traces created by the agents not in the environment, but in a representation thereof $-a$ map (see [20]).

Consider the following example: if a plurality of agents, having their own means of localization (e.g. GPS), periodically report their current position to a central station, then it is possible to create at the base station a data structure embedding geographical, topological and virtual pheromone information for the considered space (see Figure 5).

Basically, this is a Cartesian 2D grid map, where the agents mark their trajectories by placing a discrete pheromone source in each cell of the map they cross.

Just like the biological pheromones, virtual pheromones diffuse in space up to a maximum distance $\sigma$, and their effect decreases with time through evaporation, so that unreinforced traces eventually disappear.

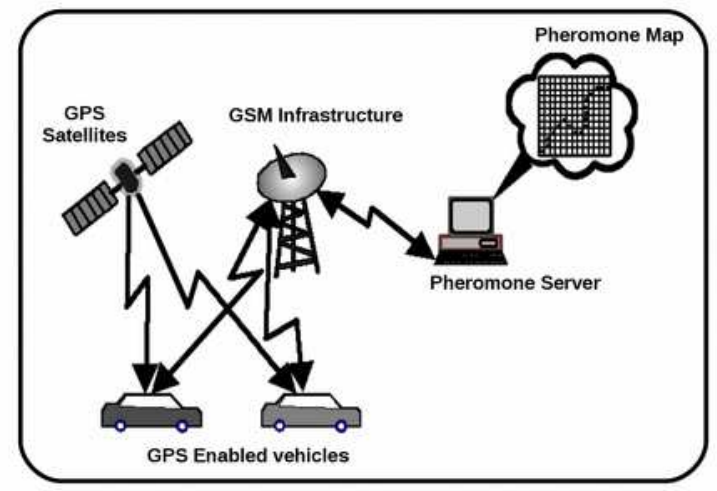

Figure 5. An example of a technological set-up for creating global cognitive maps based on virtual pheromones

Assuming a simple, linear model of diffusion, a discrete source of pheromones $S_{k}$, having the intensity $P_{k}$, can be sensed at a certain distance $\mathrm{x}$ with the intensity:

$$
p(x)=\left\{\begin{array}{cc}
p_{k}\left(1-\frac{x}{\sigma}\right) & 0<x<\sigma \\
0 & x \geq \sigma
\end{array}\right.
$$

The superposition of the effects of all $\mathrm{N}$ sources leads to:
$P_{R}=\sum_{k=1}^{N} p_{k}\left(1-\frac{d_{k}}{\sigma}\right)$

And, considering the evaporation (we also assume here a linear model for the sake of simplicity), the resulting pheromone intensity sensed in an arbitrary point is:

$$
P_{R}(t)=\sum_{k=1}^{N} p_{k}\left(1-\frac{d_{k}}{\sigma}\right)\left(1-\frac{t-t_{k}}{\tau}\right)
$$

where $t_{k}$ is the "birth moment timestamp" of the source $S_{k}$, and, $\tau$ is an evaporation constant.

If the map that embeds the information about the distribution of virtual pheromones is shared with all the agents (as a common memory), they acquire a global knowledge about the environment, which allows them to adjust their behaviour considering the activity of the other agents. For example, the system presented in Figure 5 has been the starting point of the invention described in [21], which refers to a vehicle navigation system capable to induce "ant colony optimization" processes in the road traffic.

As Parunak noted in [17], the "space" representing the MAS environment is not necessarily a Cartesian space. Graphs (hierarchical or not), or just lists (databases) of items, can equally be considered as environments capable to "host" selforganization processes.

The simple model of virtual pheromones, described by (3) does not require the absolute positions of the agent and the pheromone sources - only the relative distances between the agents and the pheromone sources matter. Thus, the model of virtual pheromones proposed above is applicable in other metric spaces, provided that a distance function can be defined.

We have described in [23] a MAS experiment wherein the environment is a large set of learning objects $\Lambda$, organized as a database that can be independently accessed by a number of users (the agents), who can search and retrieve learning objects .

For $\forall x \in \Lambda$ there exists a set keyword tags:

$$
K_{x}=\left\{k_{1}, k_{2}, \ldots, k_{\mu x}\right\}
$$

where $\mu_{x}=\left|K_{x}\right|$ is the cardinal of the set $K_{x}$.

For any two elements $x_{i}, x_{j} \in \Lambda$ it is possible to define the relative distance between them: 
$d_{i j}=1-S_{i j}$

where $S_{i j}$ is the Jaccard similarity index:

$$
S_{i j}=\frac{\left|K_{i} \cap K_{j}\right|}{\left|K_{i} \cup K_{j}\right|}
$$

It is possible to attach to each element $x_{j}$ of $\Lambda$ a numeric value $P_{j}$ (the virtual pheromone), and having the distance calculated with (5) - we can describe the effect of diffusion. Whenever the element $x_{j}$ is accessed, the value of $P_{j}$ is increased with a constant ratio $P_{j}=P_{j}+\delta p$, and the virtual pheromone source associated with $P_{j}$ diffuse to all other elements, so that for any $x_{i} \in \Lambda: i \neq j$ :

$$
P_{i}=P_{i}+P_{j}\left(1-\frac{d_{i j}}{\sigma}\right)
$$

The effect of evaporation can be easily modelled by decreasing at constant time intervals the pheromone value of all the items in the database.

The resulting virtual pheromone intensities can serve two purposes: first, as a simple way to implement a basic recommender system (items having high pheromone values can be presented as more desirable), and secondly, as a means to automatically purge large databases of unused items in a process somewhat similar to the apoptosis described in biology.

Other examples of self-organization induced by virtual pheromones are described in [22], and [24].

\subsection{The behaviour is the message}

The idea of creating global cognitive maps of the common environment in MAS, as in the examples described above, can be criticized for the centralist approach, which lacks the inherent robustness of the distributed systems.
It may be true, but this disadvantage is compensated by several major advantages, the most important of which being the capability to capture "Behavioural Implicit Communication" (BIC) messages ([17]).

As emphasized in [4], BIC should not be confused with "non-verbal communication", or "expressive behaviour". For the purpose of this presentation, we will use the term BIC to designate the process of attaching (at the level of the observer) a "meaning" to observed actions (see also [27]).

When a person chooses to drive on a particular road, to buy a product, to visit a web page, or simply to be present in a certain place at a certain time, he involuntarily sends a message about a personal preference, attitude, or value. If an observer is able to decode all the BIC messages generated in a community of agents, and integrate them in a data structure that can be shared with all the agents, this may influence the behaviour of the other agents, and create stigmergic effects.

For example, in the system shown in Figure 5, if the pheromone distribution map is made available for all the agents, the drivers obtain real-time information about how practicable are the roads in a certain geographic area, starting from the global cognitive map resulted from observing the behaviour of the other agents.

Again, in the example of the database with shared learning objects, the pheromone based cognitive map also embeds BIC messages (the implicit preference for certain LO).

By analysing the above examples (and those presented in [22], and [24]) it is possible to derive a general structure for systems capable to generate stigmergic interactions by means of global cognitive maps, as shown in Figure 6.

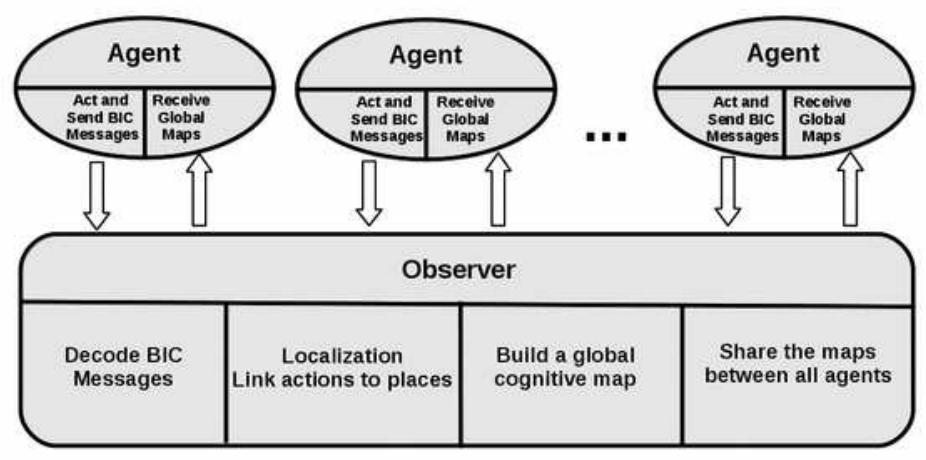

Figure 6. The general structure of stigmergic system with human agents 
Unlike the general stigmergic system presented in Figure 1, the proposed structure does not consider the traces created by the agents in the environment, but the reflection of their activity in a global cognitive map created by an observer, and shared with all the agents.

Obviously, the task of implementing the observer of a system with the structure presented in Figure 6 is not trivial. This is actually the key for creating technological environments to foster stigmergic interactions between human agents.

\subsection{Towards automatic generation of cognitive maps starting from BIC messages}

In some cases, being in a certain place, as in the example presented in Figure 5, is in itself an easy way to decode BIC message. However, in most cases, the problem of automatic recognition of certain human behaviours is much more difficult. There are literally thousands of studies, and dozens of significant approaches to the problem of recognizing a particular abnormal behaviour: fall detection ([13]). And, of course, the topics related to activity recognition are far more complex than fall detection.

The problem of linking behaviours with places (localization) is equally difficult (see for example [1], [12], and [26] for a flavour of the complexity of this problem. ).

Fortunately, in most cases, the interactions between human agents in large communities are technologically mediated, and the behaviours that must be recognized as carriers of BIC messages are just particular uses of the technological gadgets, which are now part of the everyday life environment.

The localization is also simplified by the technological context of actions, and - in many cases - the kind of localization we need for creating global cognitive maps is not necessarily a set of Cartesian coordinates, but rather to find a category that may include the agent that generates a certain BIC message (e.g. in a stigmergic marketing system, the age of the target user may be more important than the place where he lives).

In what concerns the actual generation of the global cognitive maps, the concept of virtual pheromones defined in subsection 3.1 offers a generic, and easy to use solution.
It appears that the general model of a stigmergic system for intelligent agents presented here is relatively easy to implement in a large variety of practical applications.

\section{Conclusions and Further Work}

In this paper we have explored the problem of technologically mediated indirect coordination of intelligent agents.

Unlike the biological stigmergic systems, the proposed solution does not consider the traces created by the agents in the environment, but the reflection of their activity in a global cognitive map created by an observer, and shared with all the agents.

Several practical use cases presented in [22] and [24] suggest that this approach may be the core for a variety of interesting applications.

Further research is needed to integrate in our model elements of cortically inspired computation ([1]), and to explore an alternative distributed implementation of the observer, based on the concept of "active environment" ([6]), and, of course, to identify more applications of the proposed structure, mainly in what concerns improving collaborative idea generation ([15]).

\section{Acknowledgements}

The authors would like to express their gratitude to Prof. Jörg Conradt, of the Neuroscientific System Theory Group, Technical University of Munich, whose challenging ideas about distributed cognitive maps, and "place agents" ([6]) motivated us to write this article.

\section{REFERENCES}

1. AXENIE, C., J. CONRADT, Cortically Inspired Sensor Fusion Network for Mobile Robot Egomotion Estimation. Robotics and Autonomous Systems, 2014.

2. BENI, G., J. WANG, Swarm Intelligence in Cellular Robotic Systems. In: Robots and Biological Systems: Towards a New Bionics?, Springer Berlin Heidelberg, 1993. pp. 703-712.

3. BONABEAU, E., M. DORIGO, G. THERAULAZ, Swarm Intelligence: 
from Natural to Artificial Systems. Oxford University Press, 1999.

4. CASTELFRANCHI, Cristiano. Silent Agents: From Observation to Tacit Communication. In: Advances in Artificial Intelligence-IBERAMIA-SBIA 2006. Springer Berlin Heidelberg, 2006. pp. 98-107.

5. CHIALVO, D. R., M. M. MILlONAS, How Swarms Build Cognitive Maps. In: The biology and technology of intelligent autonomous agents. Springer Berlin Heidelberg, 1995. pp. 439-450.

6. CONRADT, J.-A., A Distributed Cognitive Map for Spatial Navigation based on Graphically Organized Place Agents. 2008. PhD Thesis. Swiss Federal Institute of Technology, Zurich

7. DORIGO, M. (ed.), Ant Colony Optimization and Swarm Intelligence: 5th International Workshop, ANTS 2006, Brussels, Belgium, September 4-7, 2006, Proceedings. Springer Science \& Business Media, 2006.

8. DUNBAR, R. IM., Coevolution of Neocortical Size, Group Size and Language in Humans. Behavioural and brain sciences, 1993, 16.04: 681-694.

9. GRASSÉ, P.-P., La reconstruction du nid et les coordinations interindividuelles chez Bellicositermes natalensis et Cubitermes sp. La théorie de la stigmergie: Essai d'interprétation du comportement des termites constructeurs. Insectes sociaux, vol. 6.1, 1959, pp. 41-80.

10. HEYLIGHEN, F., Stigmergy as a Universal Coordination Mechanism: Components, Varieties and Applications. Human Stigmergy: Theoretical Developments and New Applications. Springer. Retrieved from http://pespmc1.vub.ac.be/papers/stigmergyvarieties.pdf, 2015.

11. KARLSON, P., M. LÜSCHER, 'Pheromones': a New Term for a Class of Biologically Active Substances. Nature, vol. 183,1959 , pp. 55-56.

12. LESTER, J., T. CHOUDHURY, G. BORRIELlO, A Practical Approach to Recognizing Physical Activities. In:
Pervasive Computing. Springer Berlin Heidelberg, 2006. pp. 1-16.

13. MUBASHIR, M., L. SHAO, L., SEED, A Survey on Fall Detection: Principles and Approaches. Neurocomputing, vol. 100, 2013, pp. 144-152.

14. NEGULESCU, S. C., I. DZITAC, A. E. LASCU, Synthetic Genes for Artificial Ants. Diversity in Ant Colony Optimization Algorithms. International Journal of Computers Communications \& Control, vol. 5(2), 2010, pp. 216-223.

15. NICULESCU, A., G. THORSTEINSSON, Enabling Idea Generation through Computer-Assisted Collaborative Learning. Studies in Informatics and Control, vol. 20(4), 2011, pp. 403-410.

16. O'KEEFE, J., J. DOSTROVSKY, The Hippocampus as a Spatial Map. Preliminary Evidence from Unit Activity in the Freely-moving Rat. Brain Research, vol, 34(1), 1971, pp. 171-175.

17. OMICINI, A. et al. A Conceptual Framework for Self-Organising MAS. In: WOA. 2004, pp. 100-109.

18. PARUNAK, H. V. D., A Survey of Environments and Mechanisms for Human-Human Stigmergy. In: Environments for Multi-Agent Systems II. Springer Berlin Heidelberg, 2006. pp. 163-186.

19. PARUNAK, H. V. D., S. A. BRUECKNER, The Cognitive Aptitude of Swarming Agents. Vector Research Center, Ann Arbor, MI, 2009.

20. SUSNEA, I. et al. Virtual Pheromones for Real-time Control of Autonomous Mobile Robots. Studies in Informatics and Control, vol. 18(3), 2009, pp. 233-240.

21. SUSNEA, I., G. VASILIU, Patent application "Sistem de navigatie pentru vehicule" (Vehicle Navigation System), Romanian National Patent Office OSIM Application number A00960/11.10.2010

22. SUSNEA, I., Applications of the Emergence in Cognitive MAS, The Annals of the University "Dunarea de Jos", Vol 35 (2), 2012

23. SUSNEA, I., G. VASILIU, D. E. MITU, Enabling Self-Organization of the Educational Content in Ad Hoc Learning Networks. Studies in 
Informatics and Control, vol. 22(2), 2013, pp. 143-152.

24. SUSNEA, I., Engineering Human Stigmergy, in press (to appear in International Journal of Computers Communication and Control ISSN 18419836, vol. 10 (3), June, 2015.

25. TOLMAN, E. C. Cognitive Maps in Rats and Men. Psychological Review, vol. 55(4), 1948, p. 189.
26. THRUN, S. et al. Robotic Mapping: A Survey. Exploring artificial intelligence in the new millennium, 2002, pp. 1-35.

27. TUMMOLINI, L., C. CASTELFRANCHI, Trace Signals: The Meanings of Stigmergy. In: Environments for multiagent systems III. Springer Berlin Heidelberg, 2007. p. 141-156. 